\title{
Direct detection of fourth generation Majorana neutrino dark matter
}

\author{
Yu-Feng Zhou* \\ State Key Laboratory of Theoretical Physics, \\ Kavli Institute for Theoretical Physics China, \\ Institute of Theoretical Physics, Chinese Academy of Sciences, \\ Beijing, 100190, P.R. China \\ E-mail: yfzhoueitp.ac.cn
}

\begin{abstract}
Heavy stable fourth generation Majorana neutrinos contribute to a small fraction of the relic density of dark matter (DM) in the Universe. Due to its relatively strong coupling to the standard model particles, it can be probed by the current direct and indirect DM detection experiments even it is a subdominant component of the halo DM. We show that the current Xenon100 data constrain the mass of the stable Majorana neutrino to be greater than the mass of the top quark. The effective spin-independent cross section for the neutrino elastic scattering off nucleon is predicted to be $\sim 1.5 \times 10^{-44} \mathrm{~cm}^{2}$, which is insensitive to the neutrino mass and mixing and can be reached by the direct $\mathrm{DM}$ detection experiments in the near future. In the same mass region the predicted effective spin-dependent cross section for the heavy neutrino scattering off proton is in the range of $2 \times 10^{-40} \mathrm{~cm}^{2} \sim 2 \times 10^{-39} \mathrm{~cm}^{2}$, which is within the reach of the ongoing DM indirect search experiments. We demonstrate such properties of the heavy neutrino DM in a fourth generation model with the stability of the fourth Majorana neutrino protected by an anomaly-free $U(1)$ gauge symmetry.
\end{abstract}

VIII International Workshop on the Dark Side of the Universe,

June 10-15, 2012

Rio de Janeiro, Brazil

\footnotetext{
*Speaker.
} 
Models with chiral fourth generation fermions are among the simplest and well-motivated extensions of the standard model (SM) and have been extensively studied [1]. The condition for $\mathrm{CP}$ symmetry violation in the SM requires at least three generations of fermions [2]. But, there is no upper limit on the number of generations from the first principle. In the SM the amount of $\mathrm{CP}$ violation is not large enough to explain the baryon-antibaryon asymmetry in the Universe. The inclusion of fourth generation quarks leads to two extra $\mathrm{CP}$ phases in quark sector and possible larger CP violation [3], which is helpful for electroweak baryogenesis. With very massive quarks in the fourth generation, it has been proposed that the electroweak symmetry breaking may become a dynamical feature of the SM [4-7].

Heavy stable neutrinos with mass greater than $\sim 1 \mathrm{GeV}$ are possible candidates for the cold DM $[8,9]$. However, if the neutrino is the dominant component of the halo DM, the current DM direct search experiments have imposed strong constraints on its mass [10-15]. On the other hand, it is well-known that for a neutrino heavier than $\sim m_{Z} / 2$, the cross section for its annihilation is in general too large to reproduce the observed DM relic density. For the neutrino heavier than $m_{W}$, the contribution from $f \bar{f}$ channels decrease rapidly, but other channels such as $W^{ \pm} W^{\mp}, Z^{0} h^{0}$ etc. are opened. For these processes the corresponding cross section does not decrease with the increasing of the neutrino mass, resulting in a relic density always decreases with the growing of the neutrino mass, and a thermal relic density far below the observed total DM relic density [16]. Thus the neutrino DM can only contribute to a small fraction of the relic density of DM and a small fraction of the halo DM density as well. Despite its very low number density in the halo, it can still be probed by the underground DM direct detection experiments due to its relatively strong coupling to the target nuclei, which provides a way to search for new physics beyond the SM complementary to the LHC.

In this talk, we discuss the consequence of this possibility in a model with a fourth generation Majorana neutrino DM. The stability of the fourth Majorana neutrino protected by an additional generation-dependent $U(1)$ gauge symmetry which is anomaly-free. The details of the analysis can be found in Ref. [17]. We consider a simple extension of the SM with a sequential fourth generation and an additional $U(1)_{F}$ gauge symmetry. The $U(1)$ extensions to the SM are well motivated from the point view of grand unification such as the $S O(10)$ and $E_{6}$ and have rich phenomenology [18] which can be reached by the on going LHC experiments. The flavor contents in the model are given by

$$
q_{i L}=\left(\begin{array}{c}
u_{i L} \\
d_{i L}
\end{array}\right), \ell_{i L}=\left(\begin{array}{c}
v_{i L} \\
e_{i L}
\end{array}\right), u_{i R}, d_{i R}, v_{i R}, e_{i R}(i=1, \ldots, 4) .
$$

All the fermions in the model are vector-like under the extra gauge interactions associated with $U(1)_{F}$. The $U(1)_{F}$ charges of the fermions could be generation-dependent. In order to evade the stringent constraints from the tree-level flavor changing neutral currents (FCNCs), the $U(1)_{F}$ charges $Q_{q i}$ for the first three generation quarks are set to be the same, i.e. $Q_{q i}=Q_{q},(i=1,2,3)$ while $Q_{q 4}=-3 Q_{q}$ for the fourth generation quarks. Similarly, the $U(1)_{F}$ charges for the first three generation and the fourth generation leptons are $Q_{L}$ and $-3 Q_{L}$, respectively. In general, $Q_{q}$ and $Q_{L}$ can be different. For simplicity, we consider $Q_{q}=Q_{L}=1$. With this set of flavor contents and $U(1)_{F}$ charge assignments, it is straight forward to see that the new gauge interactions are anomaly-free. Since the gauge interaction of $U(1)_{F}$ is vector-like, the triangle anomalies of 
$\left[U(1)_{F}\right]^{3},\left[S U(3)_{C}\right]^{2} U(1)_{F}$ and $[\text { gravity }]^{2} U(1)_{F}$ are all vanishing. The anomaly of $U(1)_{Y}\left[U(1)_{F}\right]^{2}$ is zero because the $U(1)_{Y}$ hypercharges cancel for quarks and leptons separately in each generation, namely $\sum\left(-Y_{q L}+Y_{q R}\right)=0$ and $\sum\left(-Y_{\ell L}+Y_{\ell R}\right)=0$. The anomaly of $\left[S U(2)_{L}\right]^{2} U(1)_{F}$ is also zero due to the relation $\sum_{i=1}^{4} Q_{q i}=0$ and $\sum_{i=1}^{4} Q_{L i}=0$. Thus in this model, the gauge anomalies generated by the first three generation fermions are canceled by that of the fourth generation one, which also gives a motivation for the inclusion of the fourth generation.

The gauge symmetry $U(1)_{F}$ is to be spontaneously broken by the Higgs mechanism. For this purpose we introduce two SM singlet scalar fields $\phi_{a, b}$ which carry the $U(1)_{F}$ charges $Q_{a}=-2 Q_{L}$ and $Q_{b}=6 Q_{L}$ respectively. The $U(1)_{F}$ charges of $\phi_{a, b}$ are arranged such that $\phi_{a}$ can have Majorana type of Yukawa couplings to the right-handed neutrinos of the first three generations $v_{i R}(i=1,2,3)$ while $\phi_{b}$ only couples to the fourth generation neutrino $v_{4 R}$. After the spontaneous symmetry breaking, the two scalar fields obtain vacuum expectation values (VEVs) $\left\langle\phi_{a, b}\right\rangle=v_{a, b} / \sqrt{2}$. The relevant interactions in the model are given by

$$
\begin{aligned}
\mathscr{L} & =\bar{f}_{i} i \gamma^{\mu} D_{\mu} f_{i}+\left(D_{\mu} \phi_{a}\right)^{\dagger}\left(D_{\mu} \phi_{a}\right)+\left(D_{\mu} \phi_{b}\right)^{\dagger}\left(D_{\mu} \phi_{b}\right) \\
& -Y_{i j}^{d} \bar{q}_{i L} H d_{i R}-Y_{i j}^{u} \bar{q}_{i L} \tilde{H} u_{i R}-Y_{i j}^{e} \bar{\ell}_{i L} H e_{i R}-Y_{i j}^{v} \bar{\ell}_{i L} \tilde{H} v_{i R} \\
& -\frac{1}{2} Y_{i j}^{m} \overline{v_{i R}^{c}} \phi_{a} v_{j R}(i, j=1,2,3)-\frac{1}{2} Y_{4}^{m} \overline{v_{4 R}^{c}} \phi_{b} v_{4 R}-V\left(\phi_{a}, \phi_{b}, H\right)+\text { H.c. }
\end{aligned}
$$

where $f_{i}$ stand for left- and right-handed fermions, and $H$ is the SM Higgs doublet. $D_{\mu} f_{i}=$ $\left(\partial_{\mu}-i g_{1} \tau^{a} W_{\mu}^{a}-i Y g_{2} B_{\mu}-i Q_{f} g_{F} Z_{\mu}^{\prime}\right) f_{i}$ is the covariant derivative with $Z_{\mu}^{\prime}$ the extra gauge boson associated with the $U(1)_{F}$ gauge symmetry, and $g_{F}$ the corresponding gauge coupling constant. Since $\phi_{a, b}$ are SM singlets, they do not play any role in the electroweak symmetry breaking. Thus $Z^{\prime}$ obtains mass only from the VEVs of the scalars

$$
m_{Z^{\prime}}^{2}=g_{F}^{2}\left(Q_{a}^{2} v_{a}^{2}+Q_{b}^{2} v_{b}^{2}\right)
$$

From the $U(1)_{F}$ charge assignments in the model, the four by four Yukawa coupling matrix is constrained to be of the block diagonal form $3 \otimes 1$ in the generation space. Since the $U(1)_{F}$ charges are the same for the fermions in the first three generation, there is no tree level FCNC induced by the $Z^{\prime}$-exchange in the physical basis after diagonalization. Thus a number of constraints from the low energy flavor physics such as the neutral meson mixings and the $b \rightarrow s \gamma$ can be avoided.

The direct search for the process $e^{+} e^{-} \rightarrow Z^{\prime} \rightarrow \ell^{+} \ell^{-}$at the LEP-II leads to a lower bound on the ratio of the mass to the coupling to leptons: $M_{Z^{\prime}} / g_{F} \geq 6 \mathrm{TeV}$ [19] for vector-like interactions, which corresponds to a more stringent lower bound: $\sqrt{Q_{a}^{2} v_{a}^{2}+Q_{b}^{2} v_{b}^{2}} \geq 6 \mathrm{TeV}$. The current searches for narrow resonances in the Drell-Yan process $p p \rightarrow Z^{\prime} \rightarrow \ell^{+} \ell^{-}$at the LHC impose an alternative bound on the mass and the couplings of the $Z^{\prime}$ boson. For a model with sequential neutral gauge boson $Z_{S S M}^{\prime}$ which by definition has the same couplings as that for the SM $Z^{0}$ boson [18], the latest lower bounds on its mass $M_{Z_{S S M}^{\prime}}$ is $1.94 \mathrm{TeV}$ from CMS [20] and $1.83 \mathrm{TeV}$ from ATLAS [21] respectively. The bound on $M_{Z_{S S M}^{\prime}}$ can be translated into the bound on the mass and couplings of the $Z^{\prime}$ in this model. For instance, $g_{F} \lesssim 0.029$ for $M_{Z^{\prime}}=1.44 \mathrm{TeV}$ and $g_{F} \lesssim 0.0051$ for $M_{Z^{\prime}}=0.94$ $\mathrm{TeV}$, respectively.

The fourth generation neutrinos obtain both Dirac and Majorana mass terms through the vacuum expectation values (VEVs) of $H$ and $\phi_{b}$. In the basis of $\left(v_{L}, v_{R}^{c}\right)^{T}$ the mass matrix for the 
fourth neutrino is given by

$$
m_{v}=\left(\begin{array}{cc}
0 & m_{D} \\
m_{D} & m_{M}
\end{array}\right),
$$

where $m_{D}=Y_{4}^{v} v_{H} / \sqrt{2}$ with $v_{H}=246 \mathrm{GeV}$ and $m_{M}=Y_{4}^{m} v_{\phi_{b}} / \sqrt{2}$. The left-handed components $\left(v_{1 L}^{(m)}, v_{2 L}^{(m)}\right)$ of the two mass eigenstates are related to the ones in the flavor eigenstates by a rotation angle $\theta$

$$
v_{1 L}^{(m)}=-i\left(c_{\theta} v_{L}-s_{\theta} v_{R}^{c}\right), \quad v_{2 L}^{(m)}=s_{\theta} v_{L}+c_{\theta} v_{R}^{c}
$$

where $s_{\theta} \equiv \sin \theta$ and $c_{\theta} \equiv \cos \theta$. The value of $\theta$ is defined in the range $(0, \pi / 4)$ and is determined by

$$
\tan 2 \theta=\frac{2 m_{D}}{m_{M}}
$$

with $\theta=0(\pi / 4)$ corresponding to the limit of minimal (maximal) mixing. The phase $i$ is introduced to render the two mass eigenvalues real and positive. The two Majorana mass eigenstates are $\chi_{1}=v_{1 L}^{(m)}+v_{1 L}^{(m) c}$ and $\chi_{2}=v_{2 L}^{(m)}+v_{2 L}^{(m) c}$, respectively. The masses of the two neutrinos are given by $m_{1,2}=\left(\sqrt{m_{M}^{2}+4 m_{D}^{2}} \mp m_{M}\right) / 2$. In terms of the mixing angle $\theta$ they can be rewritten as $m_{1}=\left(s_{\theta} / c_{\theta}\right) m_{D}$ and $m_{2}=\left(c_{\theta} / s_{\theta}\right) m_{D}$. with $m_{1} \leq m_{2}$. Note that for all the possible values of $\theta$ the lighter neutrino mass eigenstate $\chi_{1}$ consists of more left-handed neutrino than the right-handed one, which means that $\chi_{1}$ always has sizable coupling to the SM $Z^{0}$ boson. Therefore the LEP-II bound on the mass of stable neutrino is always valid for $\chi_{1}$, which is insensitive to the mixing angle.

As the fermions in the first three generations and the fourth generation have different $U(1)_{F}$ charges, the fourth generation fermions cannot mix with the ones in the first three generations through Yukawa interactions. After the spontaneous breaking down of $U(1)_{F}$, there exists a residual $Z_{2}$ symmetry for the fourth generation fermions which protect the fourth neutrino $\chi_{1}$ to be a stable particle if it is lighter than the fourth generation charged lepton $e_{4}$, which makes it a possible dark matter candidate.

The thermal relic density of $\chi_{1}$ is related to its annihilation cross section at freeze out. We numerically calculate the cross sections for $\chi_{1} \chi_{1}$ annihilation into all the relevant final states using CalHEP 2.4 [22]. In Fig. 1, we show the quantity $r_{\Omega} \equiv \Omega_{\chi_{1}} / \Omega_{D M}$ the ratio of the relic density of $\chi_{1}$ to the observed total DM relic density $\Omega_{D M} h^{2}=0.110 \pm 0.006$ [23] as function of the mass of $\chi_{1}$ for different values of the mixing angle $\theta$. The results show a significant dependence on the mixing angle $\theta$. For smaller mixing angle $\theta$ the couplings between $\chi_{1}$ and gauge bosons $W^{ \pm}, Z$ are stronger, resulting in a smaller relic density. The results also clearly show that due to the large annihilation cross section, $\chi_{1}$ cannot make up the whole DM in the Universe. $\chi_{1}$ can contribute to $\sim 20-40 \%$ of the total DM relic density when its mass is around $80 \mathrm{GeV}$. But for $m_{1} \gtrsim m_{t}$, it can contribute only a few percent or less to the whole DM.

However, since $\chi_{1}$ has strong couplings to $h^{0}$ and $Z^{0}$, even in the case that the number density of $\chi_{1}$ is very low in the DM halo, it is still possible that it can be detected by its elastic scattering off nucleus in direct detection experiments. Given the difficulties in detecting such a neutral and 


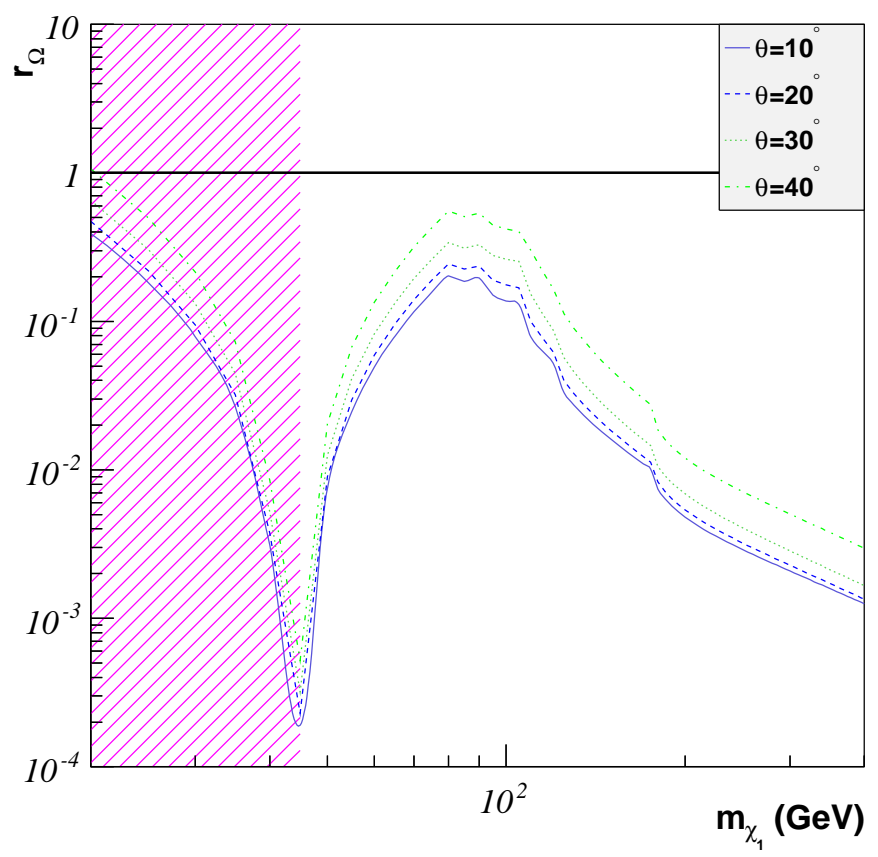

Figure 1: The rescaled $\chi_{1}$ relic density $r_{\Omega}$ as function of the mass of $\chi_{1}$. The shaded region is excluded by the LEP-II experiments.

stable particle at the LHC, there is a possibility that the stable fourth generation neutrino could be first seen at the DM direct detection experiments.

The generic formula for the differential event rate of DM-nucleus scattering per nucleus mass is given by

$$
\frac{d N}{d E_{R}}=\frac{\rho_{D M} \sigma_{N}}{2 m_{D M} \mu_{N}^{2}} F^{2}\left(E_{R}\right) \int_{v_{\min }}^{v_{e s c}} d^{3} v \frac{f(v)}{v}
$$

where $E_{R}$ is the recoil energy, $\sigma_{N}$ is the scattering cross section corresponding to the zero momentum transfer, $m_{D M}$ is the mass of the DM particle, $\mu_{N}=m_{D M} m_{N} /\left(m_{D M}+m_{N}\right)$ is the DM-nucleus reduced mass, $F\left(E_{R}\right)$ is the form factor, and $f(v)$ is the velocity distribution function of the halo DM. The local DM density $\rho_{D M}$ is often set to be equal to $\rho_{0} \simeq 0.3 \mathrm{GeV} / \mathrm{cm}^{3}$ which is the local DM density inferred from astrophysics based on a smooth halo profile. Since the neutrino DM can only contribute to a small fraction of the relic density of DM, it is likely that it also contributes to a small fraction of the halo DM density, namely, its local density $\rho_{1}$ is much smaller than $\rho_{0}$. We assume that $\rho_{1}$ is proportional to the relic density of $\chi_{1}$ in the Universe, namely

$$
r_{\rho} \equiv \frac{\rho_{1}}{\rho_{0}} \approx \frac{\Omega_{\chi_{1}}}{\Omega_{D M}}
$$

or $r_{\rho} \approx r_{\Omega}$. Consequently, the expected event rates of the DM-nucleus elastic scattering will be scaled down by $r_{\rho}$. In order to directly compare the theoretical predictions with the reported experimental upper limits which are often obtained under the assumption that the local DM particle 
density is $\rho_{0}$, we shall calculate the rescaled elastic scattering cross section

$$
\tilde{\sigma} \equiv r_{\rho} \sigma \approx r_{\Omega} \sigma
$$

which corresponds to the event rate to be seen at the direct detection experiments. Note that $\tilde{\sigma}$ depends on the mass of $\chi_{1}$ through the ratio $r_{\rho}$ even when $\sigma$ is mass-independent. The spinindependent DM-nucleon elastic scattering cross section in the limit of zero momentum transfer is given by [24]

$$
\sigma_{n}^{S I}=\frac{4 \mu_{n}^{2}}{\pi} \frac{\left[Z f_{p}+(A-Z) f_{n}\right]^{2}}{A^{2}}
$$

where $Z$ and $A-Z$ are the number of protons and neutrons within the target nucleus, respectively. $\mu_{n}=m_{1} m_{n} /\left(m_{1}+m_{n}\right)$ is the DM-nucleon reduced mass. The couplings between DM and the proton (neutron) read

$$
f_{p(n)}=\sum_{q=u, d, s} f_{T q}^{p(n)} a_{q} \frac{m_{p(n)}}{m_{q}}+\frac{2}{27} f_{T G}^{p(n)} \sum_{q=c, b, t} a_{q} \frac{m_{p(n)}}{m_{q}},
$$

with $f_{T q}^{p(n)}$ the DM coupling to light quarks and $f_{T G}^{p(n)}=1-\sum_{q=u, d, s} f_{T q}^{p(n)}$. The coefficient $a_{q}$ in the model is given by

$$
a_{q}=c_{\theta}^{2} \frac{m_{1} m_{q}}{v_{H}^{2} m_{h}^{2}}
$$

The value of $a_{q}$ is proportional to $m_{1}$, thus larger elastic scattering cross section is expected for heavier $\chi_{1}$. The quark mass $m_{q}$ in the expression of $a_{q}$ cancels the one in the expression of $f_{p(n)}$. Thus there is no quark mass dependence in the calculations.

In Fig. 2 we give the predicted spin-independent effective cross sections $\tilde{\sigma}_{n}^{S I}$ for the fourth generation neutrino elastic scattering off nucleon as function of its mass for different values of the mixing angle $\theta$. One sees that even after the inclusion of the rescaling factor $r_{\rho}$, the current Xenon 100 data can still rule out a stable fourth generation neutrino in the mass range $55 \mathrm{GeV} \lesssim$ $m_{1} \lesssim 175 \mathrm{GeV}$ which corresponds to $r_{\Omega} \lesssim 1 \%$. Thus the stable fourth generation neutrino must be heavier than the top quark, and can only contribute to a small fraction of the total DM relic density On the other hand, for $m_{\chi_{1}} \gtrsim 200 \mathrm{GeV}$, the cross section does not decrease with $m_{\chi_{1}}$ increasing, and is nearly a constant $\tilde{\sigma}_{n}^{S I} \approx 1.5 \times 10^{-44} \mathrm{~cm}^{2}$ in the range $200 \mathrm{GeV} \lesssim m_{\chi_{1}} \lesssim 400 \mathrm{GeV}$. This is due to the enhanced Yukawa coupling between the fourth generation neutrino and the Higgs boson which is proportional to $m_{\chi_{1}}$, as it is shown in the expression of $a_{q}$. Similar conclusions are expected for other models in which DM particles interact with SM particles through Higgs portal, for instance, the singlet scalar DM in extensions of left-right symmetry model [25-28]. One can see from the Fig. 2 that the result is not sensitive to the mixing angle $\theta$ either, which is due to the compensation of the similar dependencies on $\theta$ in the relic density. For instance, the cross sections for the $W^{ \pm} W^{\mp}$ and $Z^{0} Z^{0}$ channel of $\chi \chi$ annihilation are proportional to $c_{\theta}^{4}$, which compensates the $\theta$-dependence in the $a_{q}$ for the elastic scattering processes.

The Majorana neutrino DM contributes also to spin-dependent elastic scattering cross section through axial-vector interaction induced by the exchange of the $Z^{0}$ boson. At zero momentum 


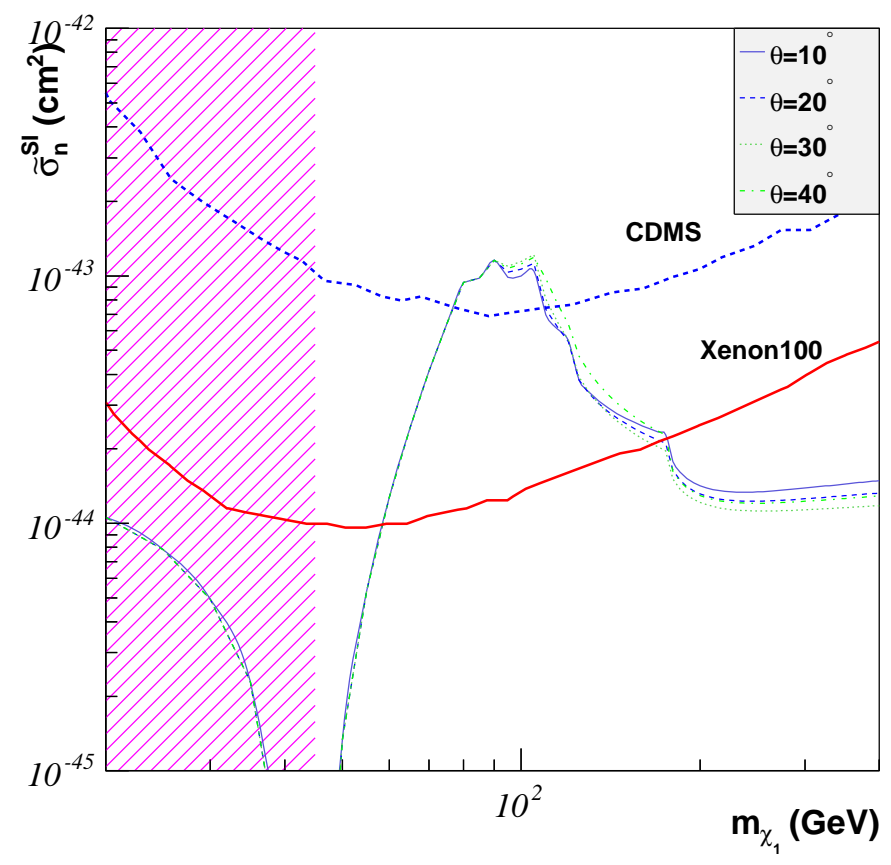

Figure 2: Effective spin-independent cross section $\tilde{\sigma}_{n}^{S I}$ which is $\sigma_{n}^{S I}$ rescaled by $r_{\rho} \approx r_{\Omega}$ for $\chi_{1}$ elastically scattering off nucleon as function of the mass of $\chi_{1}$. Four curves correspond to the mixing angle $\theta=10^{\circ}$ (solid), $20^{\circ}$ (dashed), $30^{\circ}$ (dotted) and $40^{\circ}$ (dot-dashed) respectively. The current upper limits from CDMS [29] and Xenon100 [30] experiments are also shown.

transfer, the spin-dependent cross section has the following form [24]

$$
\sigma_{N}^{S D}=\frac{32}{\pi} G_{F}^{2} \mu_{n}^{2} \frac{J+1}{J}\left(a_{p}\left\langle S_{p}\right\rangle+a_{n}\left\langle S_{n}\right\rangle\right)^{2},
$$

where $J$ is the spin of the nucleus, $a_{p(n)}$ is the DM effective coupling to proton (neutron) and $\left\langle S_{p(n)}\right\rangle$ the expectation value of the spin content of the nucleon within the nucleus. $G_{F}$ is the Fermi constant. The coupling $a_{p(n)}$ can be written as

$$
a_{p(n)}=\sum_{u, d, s} \frac{d_{q}}{\sqrt{2} G_{F}} \Delta_{q}^{p(n)},
$$

where $d_{q}$ is the DM coupling to quark and $\Delta_{q}^{p(n)}$ is the fraction of the proton (neutron) spin carried by a given quark $q$. The coefficients $d_{q}$ in this model are given by

$$
d_{u}=-d_{d}=-d_{s}=\frac{G_{F}}{\sqrt{2}} .
$$

For the axial-vector interactions, the coupling strengths do not depend on the electromagnetic charges of the quarks.

In Fig. 3 we show the predicted effective spin-dependent DM-neutron cross section $\tilde{\sigma}_{n}^{S D}$ as function of the neutrino mass for different mixing angles, together with various experimental upper 


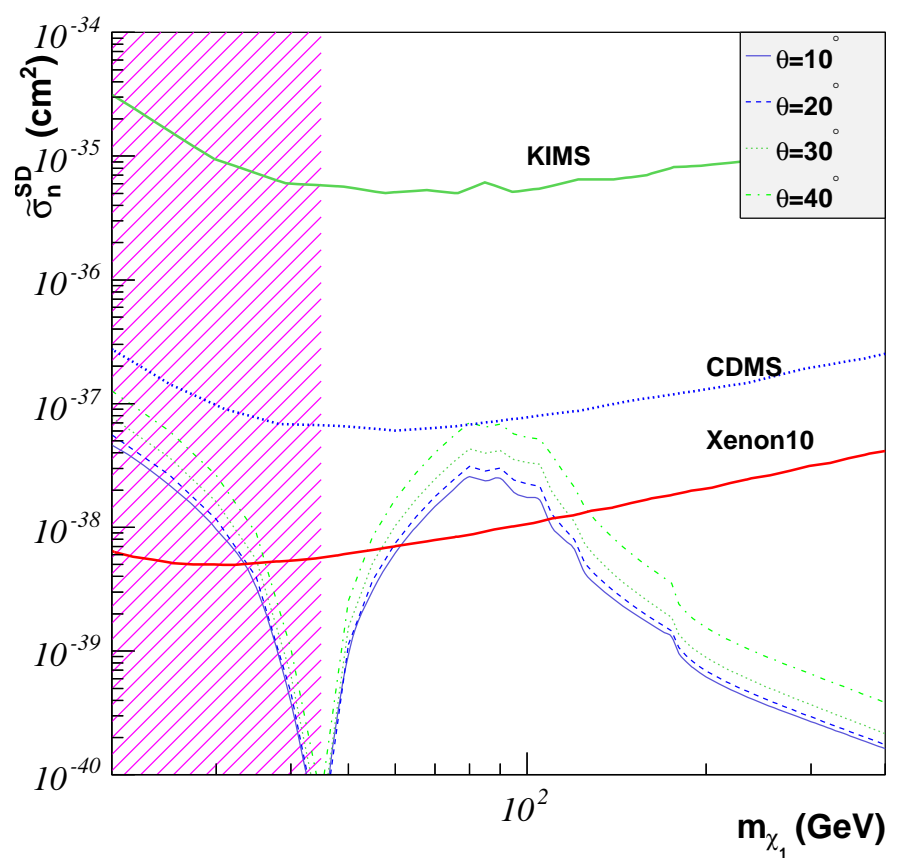

Figure 3: Effective spin-dependent cross section $\tilde{\sigma}_{n}^{S D}$ which is $\sigma_{n}^{S D}$ rescaled by $r_{\rho} \approx r_{\Omega}$ for $\chi_{1}$ elastically scattering off neutron as function of the mass of $\chi_{1}$. Four curves correspond to the mixing angle $\theta=$ $10^{\circ}$ (solid), $20^{\circ}$ (dashed), $30^{\circ}$ (dotted) and $40^{\circ}$ (dot-dashed) respectively. The current upper limits from various experiments such as KIMS [34], CDMS [35] and Xenon10 [14] are also shown.

limits. Since $\sigma_{n}^{S D}$ is independent of $m_{\chi_{1}}$, the dependency of $\tilde{\sigma}_{p(n)}^{S D}$ on the neutrino mass comes from the dependency of $r_{\rho}$ on $m_{\chi_{1}}$, which can be seen by comparing Fig. 3 with Fig. 1. The Xenon10 data is able to exclude the neutrino DM in the mass range $60 \mathrm{GeV} \lesssim m_{\chi_{1}} \lesssim 120 \mathrm{GeV}$, which is not as strong as that from the Xenon100 data on spin-independent elastic scattering cross section. For a heavy neutrino DM with mass in the range $200 \mathrm{GeV} \lesssim m_{\chi_{1}} \lesssim 400 \mathrm{GeV}$ the predicted spin-dependent cross section is between $10^{-40} \mathrm{~cm}^{2}$ and $10^{-39} \mathrm{~cm}^{2}$.

In Fig. 4 we give the predicted spin-dependent DM-proton cross section $\tilde{\sigma}_{p}^{S D}$. The cross sections for Majorana neutrino DM scattering off proton and neutron are quite similar, which is due to the fact that the relative opposite signs in $\Delta_{u}$ and $\Delta_{d}$ are compensated by the opposite signs in $d_{u}$ and $d_{n}$. So far the most stringent limit on the DM-proton spin-dependent cross section is reported by the SIMPLE experiment [31]. The SIMPLE result is able to exclude the mass range $50 \mathrm{GeV} \lesssim m_{\chi_{1}} \lesssim 150 \mathrm{GeV}$, which is compatible with the constraints from Xenon100. In Fig. 4, we also show the upper limits from indirect searches using up-going muons which are related to the annihilation of stable fourth generation neutrinos captured in the Sun. The limit from the Super-K experiment is obtained with the assumption that $80 \%$ of the DM annihilation products are from $b \bar{b}$, $10 \%$ from $c \bar{c}$ and $10 \%$ from $\tau \bar{\tau}$ respectively [32]. In the range $170 \mathrm{GeV} \lesssim m_{\chi_{1}} \lesssim 400 \mathrm{GeV}$, the limit from Super-K is $\sim 5 \times 10^{-39} \mathrm{~cm}^{2}$. The IceCube sets a stronger limit $\tilde{\sigma}_{p}^{S D} \leq 2 \times 10^{-40} \mathrm{~cm}^{2}$ for the DM mass at $250 \mathrm{GeV}$ [33]. This limit is obtained with the assumption that the DM annihilation 


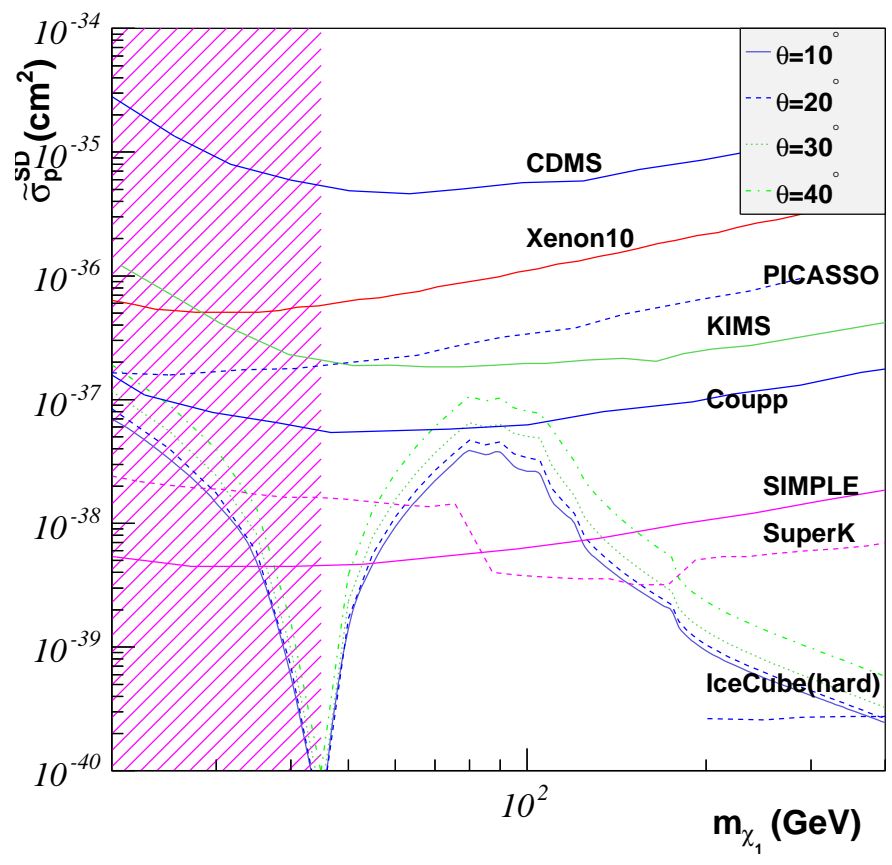

Figure 4: Effective spin-dependent cross section $\tilde{\sigma}_{p}^{S D}$ which is $\sigma_{p}^{S D}$ rescaled by $r_{\rho} \approx r_{\Omega}$ for $\chi_{1}$ elastically scattering off proton as function of the mass of $\chi_{1}$. Four curves correspond to the mixing angle $\theta=10^{\circ}$ (solid), $20^{\circ}$ (dashed), $30^{\circ}$ (dotted) and $40^{\circ}$ (dot-dashed) respectively. The current upper limits from various experiments such as KIMS [34], CDMS [35], Xenon10 [14], Coupp [36], Picasso [37], SIMPLE [38], SuperK [32], and IceCube [33] are also shown.

products are dominated by $W^{ \pm} W^{\mp}$. If the annihilation products are dominated by $b \bar{b}$, the limit is much weaker, for instance $\tilde{\sigma}_{p}^{S D} \leq 5 \times 10^{-38} \mathrm{~cm}^{2}$ for the DM mass at $500 \mathrm{GeV}$ [33]. Note that in this model, the dominant final state is $Z^{0} h^{0}$. The expected limit should be somewhere in between. Nevertheless, the IceCube has the potential to test these predictions.

In summary, we have investigated the properties of stable fourth generation Majorana neutrinos as dark matter particles. Although they contribute to a small fraction of the whole DM in the Universe, they can still be easily probed by the current direct detection experiments due to their relatively strong couplings to the SM particles. We have considered a fourth generation model with the stability of the fourth Majorana neutrino protected by an additional generation-dependent $U(1)$ gauge symmetry. We have shown that the current Xenon100 data constrain the mass of the stable Majorana neutrino to be greater than the mass of the top quark. For a stable Majorana neutrino heavier than the top quark, the effective spin-independent cross section for the elastic scattering off nucleon is found to be insensitive to the neutrino mass and is predicted to be around $10^{-44} \mathrm{~cm}^{2}$, which can be reached by the direct DM search experiments in the near future. The predicted effective spin-dependent cross section for the heavy neutrino scattering off proton is in the range $10^{-40} \mathrm{~cm}^{2} \sim 10^{-39} \mathrm{~cm}^{2}$, which can be tested by the ongoing DM indirect search experiments such as IceCube. This work is supported in part by the National Basic Research Program of China (973 
Program) under Grants No. 2010CB833000; the National Nature Science Foundation of China (NSFC) under Grants No. 10975170, No. 10821504 and No. 10905084; and the Project of Knowledge Innovation Program (PKIP) of the Chinese Academy of Science.

\section{References}

[1] P. H. Frampton, P. Q. Hung, and M. Sher, Quarks and leptons beyond the third generation, Phys. Rept. 330 (2000) 263, [hep-ph / 9903387$].$

[2] M. Kobayashi and T. Maskawa, CP Violation in the Renormalizable Theory of Weak Interaction, Prog. Theor. Phys. 49 (1973) 652-657.

[3] W.-S. Hou, CP Violation and Baryogenesis from New Heavy Quarks, Chin. J. Phys. 47 (2009) 134, [arXiv:0803.1234].

[4] B. Holdom, heavy quarks and electroweak symmetry breaking, Phys. Rev. Lett. 57 (1986) 2496.

[5] J. Carpenter, R. Norton, S. Siegemund-Broka, and A. Soni, properties of a composite higgs particle in a dynamically broken formulation of the standard model, Phys. Rev. Lett. 65 (1990) 153-156.

[6] C. T. Hill, M. A. Luty, and E. A. Paschos, Electroweak symmetry breaking by fourth generation condensates and the neutrino spectrum, Phys.Rev. D43 (1991) 3011-3025.

[7] P. Q. Hung and C. Xiong, Renormalization Group Fixed Point with a Fourth Generation: Higgs-induced Bound States and Condensates, Nucl. Phys. B847 (2011) 160-178, [arXiv:0911.3890].

[8] B. W. Lee and S. Weinberg, Cosmological lower bound on heavy-neutrino masses, Phys. Rev. Lett. 39 (1977) 165-168.

[9] E. W. Kolb and K. A. Olive, The Lee-Weinberg Bound Revisited, Phys. Rev. D33 (1986) 1202. [Erratum-ibid.D34:2531,1986].

[10] M. W. Goodman and E. Witten, Detectability of certain dark-matter candidates, Phys. Rev. D31 (1985) 3059.

[11] M. Srednicki, K. A. Olive, and J. Silk, High-Energy Neutrinos from the Sun and Cold Dark Matter, Nucl. Phys. B279 (1987) 804.

[12] T. Falk, K. A. Olive, and M. Srednicki, Heavy Sneutrinos as Dark Matter, Phys. Lett. B339 (1994) 248-251, [hep-ph/9409270].

[13] G. Belanger, A. Pukhov, and G. Servant, Dirac Neutrino Dark Matter, JCAP 0801 (2008) 009, [arXiv:0706.0526].

[14] J. Angle et. al., Limits on spin-dependent WIMP-nucleon cross-sections from the XENON10 experiment, Phys. Rev. Lett. 101 (2008) 091301, [arXiv: 0805.2939].

[15] W.-Y. Keung and P. Schwaller, Long Lived Fourth Generation and the Higgs, JHEP 06 (2011) 054, [arXiv: 1103.3765].

[16] K. Enqvist, K. Kainulainen, and J. Maalampi, cosmic abundances of very heavy neutrinos, Nucl. Phys. B317 (1989) 647-664.

[17] Y.-F. Zhou, Probing the fourth generation Majorana neutrino dark matter, Phys.Rev. D85 (2012) 053005, [arXiv: 1110.2930]. 
[18] P. Langacker, The Physics of Heavy Z-prime Gauge Bosons, Rev. Mod. Phys. 81 (2009) 1199-1228, [arXiv:0801.1345].

[19] M. S. Carena, A. Daleo, B. A. Dobrescu, and T. M. P. Tait, Z-prime gauge bosons at the Tevatron, Phys. Rev. D70 (2004) 093009, [hep-ph / 0408098$].$

[20] for the CMS Collaboration, V. Timciuc, Search for High-Mass Resonances in the Dilepton Final State with the CMS Detector, arXiv: 1111.4528.

[21] A. Collaboration, Search for dilepton resonances in pp collisions at sqrt(s) = 7 TeV with the ATLAS detector, Phys. Rev. Lett. 107 (2011) 272002, [arXiv: 1108 . 1582].

[22] G. Belanger et. al., Indirect search for dark matter with micrOMEGAs2.4, Comput. Phys. Commun. 182 (2011) 842-856, [arXiv: 1004 .1092].

[23] Particle Data Group Collaboration, K. Nakamura et. al., Review of particle physics, J. Phys. G37 (2010) 075021.

[24] G. Jungman, M. Kamionkowski, and K. Griest, Supersymmetric dark matter, Phys.Rept. 267 (1996) 195-373, [hep-ph/9506380].

[25] W.-L. Guo, Y.-L. Wu, and Y.-F. Zhou, Dark matter candidates in left-right symmetric models, Int.J.Mod.Phys. D20 (2011) 1389-1397.

[26] W.-L. Guo, Y.-L. Wu, and Y.-F. Zhou, Searching for Dark Matter Signals in the Left-Right Symmetric Gauge Model with CP Symmetry, Phys.Rev. D82 (2010) 095004, [arXiv: 1008 . 44 79].

[27] W.-L. Guo, Y.-L. Wu, and Y.-F. Zhou, Exploration of decaying dark matter in a left-right symmetric model, Phys.Rev. D81 (2010) 075014, [arXiv: 1001 . 0307].

[28] W.-L. Guo, L.-M. Wang, Y.-L. Wu, Y.-F. Zhou, and C. Zhuang, Gauge-singlet dark matter in a left-right symmetric model with spontaneous CP violation, Phys.Rev. D79 (2009) 055015 , [arXiv:0811.2556].

[29] The CDMS-II Collaboration Collaboration, Z. Ahmed et. al., Dark Matter Search Results from the CDMS II Experiment, Science 327 (2010) 1619-1621, [arXiv: 0912 . 3592].

[30] XENON100 Collaboration Collaboration, E. Aprile et. al., Dark Matter Results from 100 Live Days of XENON100 Data, Phys.Rev.Lett. (2011) [arXiv: 1104 . 2549 ].

[31] M. Felizardo et. al., Final Analysis and Results of the Phase II SIMPLE Dark Matter Search, arXiv:1106.3014.

[32] Super-Kamiokande Collaboration, S. Desai et. al., Search for dark matter WIMPs using upward through-going muons in Super-Kamiokande, Phys. Rev. D70 (2004) 083523, [hep-ex/ 0404025 ]. [Erratum-ibid.D70:109901,2004].

[33] ICECUBE Collaboration, R. Abbasi et. al., Limits on a muon flux from neutralino annihilations in the Sun with the IceCube 22-string detector, Phys. Rev. Lett. 102 (2009) 201302, [arXiv: 0902 . 2460 ].

[34] KIMS Collaboration, H. S. Lee et. al., Limits on WIMP-nucleon cross section with CsI(Tl) crystal detectors, Phys. Rev. Lett. 99 (2007) 091301, [arXiv: 0704 . 0423].

[35] CDMS Collaboration, D. S. Akerib et. al., Limits on spin-dependent WIMP nucleon interactions from the Cryogenic Dark Matter Search, Phys. Rev. D73 (2006) 011102, [astro-ph / 0509269 ].

[36] E. Behnke et. al., Improved Limits on Spin-Dependent WIMP-Proton Interactions from a Two Liter $\mathrm{CF}_{3} I$ Bubble Chamber, Phys. Rev. Lett. 106 (2011) 021303, [arXiv: 1008 . 3518]. 
[37] S. Archambault et. al., Dark Matter Spin-Dependent Limits for WIMP Interactions on 19-F by PICASSO, Phys. Lett. B682 (2009) 185-192, [arXiv: 0907 . 0307].

[38] M. Felizardo, T. Girard, T. Morlat, A. Fernandes, F. Giuliani, et. al., Final Analysis and Results of the Phase II SIMPLE Dark Matter Search, arXiv:1106.3014. 